\title{
Improved direct and converse theorems in weighted Lorentz spaces
}

\author{
Ramazan Akgün \\ Yunus Emre Yıldırır
}

\begin{abstract}
In the present work we prove the equivalence of the fractional modulus of smoothness to the realization functional and to the Peetre K-functional in weighted Lorentz spaces. Using this equivalence we obtain an improvement of the direct approximation theorem. Furthermore we prove the improved converse theorem in this space.
\end{abstract}

\section{Introduction and main results}

In approximation theory improvements of direct and inverse theorems have been investigated by several authors in different function spaces $[1,9,12,18,20,21]$. In this paper we deal with the improved direct and inverse approximation theorems in the weighted Lorentz space $L_{\omega}^{p q}(\mathbb{T})$ with Muckenhoupt weights. To obtain the improved direct theorem we need the realization and characterization theorem in $L_{\omega}^{p q}(\mathbb{T})$. Therefore we will prove a realization result and an equivalence relation between the modulus of smoothness and the Peetre $K$-functional in $L_{\omega}^{p q}(\mathbb{T})$. Furthermore, the realization result has a lot of applications [6]. In particular, it is used to get Ul'yanov type inequalities [8]. First, we give some definitions and properties.

Let $\mathbb{T}:=[-\pi, \pi]$ and $\omega: \mathbb{T} \rightarrow[0, \infty]$ be a weight function i.e., an almost everywhere positive measurable function. We define the decreasing rearrange-

Received by the editors in June 2014 - In revised form in January 2016.

Communicated by H. De Schepper.

2010 Mathematics Subject Classification : 41A25, 41A27, 42A10.

Key words and phrases : Fractional modulus of smoothness, realization, weighted Lorentz spaces, Muckenhoupt weight, direct and converse theorem. 
ment $f_{\omega}^{*}(t)[11]$ of $f: \mathbb{T} \rightarrow \mathbb{R}$ with respect to the Borel measure

$$
\omega(e)=\int_{e} \omega(x) \mathrm{d} x
$$

by

$$
f_{\omega}^{*}(t)=\inf \{\tau \geq 0: \omega(x \in \mathbb{T}:|f(x)|>\tau) \leq t\} .
$$

The weighted Lorentz space $L_{\omega}^{p q}(\mathbb{T})$ is defined [11] as

$$
L_{\omega}^{p q}(\mathbb{T})=\left\{f \in \mathbf{M}(\mathbb{T}):\|f\|_{p q, \omega}=\left(\int_{\mathbb{T}}\left(f^{* *}(t)\right)^{q} t^{\frac{q}{p}} \frac{\mathrm{d} t}{t}\right)^{1 / q}<\infty, 1<p, q<\infty\right\},
$$

where $\mathbf{M}(\mathbb{T})$ is the set of $2 \pi$ periodic integrable functions on $\mathbb{T}$ and

$$
f^{* *}(t)=\frac{1}{t} \int_{0}^{t} f_{\omega}^{*}(u) \mathrm{d} u
$$

If $p=q, L_{\omega}^{p q}(\mathbb{T})$ turns into the weighted Lebesgue space $L_{\omega}^{p}(\mathbb{T})[11, \mathrm{p} .20]$.

A weight function $\omega: \mathbb{T} \rightarrow[0, \infty]$ belongs to the Muckenhoupt class $A_{p}$ [17], $1<p<\infty$, if

$$
\sup \frac{1}{|I|} \int_{I} \omega(x) \mathrm{d} x\left(\frac{1}{|I|} \int_{I} \omega^{1-p^{\prime}}(x) d x\right)^{p-1}=C_{A_{p}}<\infty, \quad p^{\prime}:=\frac{p}{p-1}
$$

with a finite constant $C_{A_{p}}$ independent of $I$, where the supremum is taken over all intervals $I$ with length $\leq 2 \pi$ and $|I|$ denotes the length of $I$. The constant $C_{A_{p}}$ is called the Muckenhoupt constant of $\omega$.

By the proof of [14, Prop. 3.3], we know that $L_{w}^{p q}(\mathbb{T}) \subset L^{1}(\mathbb{T})$. Let

$$
S[f]:=\sum_{k=-\infty}^{\infty} c_{k} e^{i k x}
$$

be the Fourier series of a function $f \in L^{1}(\mathbb{T})$. Assume that

$$
\int_{\mathbb{T}} f(x) \mathrm{d} x=0 .
$$

For $\alpha \in \mathbb{R}^{+}$, we define the $\alpha$-th fractional integral of $f$ as [22, v.2, p.134]

$$
I_{\alpha}(x, f):=\sum_{k \in \mathbb{Z}^{*}} c_{k}(i k)^{-\alpha} e^{i k x},
$$

with

$$
(i k)^{-\alpha}:=|k|^{-\alpha} e^{(-1 / 2) \pi i \alpha \operatorname{sign} k}
$$

as principal value.

We define the fractional derivative of a function $f \in L^{1}(\mathbb{T})$, satisfying (2), as

$$
f^{(\alpha)}(x):=\frac{\mathrm{d}^{[\alpha]+1}}{\mathrm{~d} x^{[\alpha]+1}} I_{1+\alpha-[\alpha]}(x, f)
$$


whenever the right hand side exists.

For a function $f \in L_{\omega}^{p q}(\mathbb{T}), 1<p, q<\infty, w \in A_{p}$, Steklov's mean operator is defined as

$$
\sigma_{h} f(x):=\frac{1}{2 h} \int_{x-h}^{x+h} f(u) \mathrm{d} u, \quad x \in \mathbb{T} .
$$

Whenever $\omega \in A_{p}, 1<p, q<\infty$, the Hardy-Littlewood maximal function of $f \in L_{\omega}^{p q}(\mathbb{T})$ belongs to $L_{\omega}^{p q}(\mathbb{T})\left[5\right.$, Theorem 3]. Therefore the operator $\sigma_{h} f$ belongs to $L_{\omega}^{p q}(\mathbb{T})$. Using this fact and putting $x, t \in \mathbb{T}, r \in \mathbb{R}^{+}, \omega \in A_{p}$ and $f \in L_{\omega}^{p q}(\mathbb{T})$, $1<p, q<\infty$, we define

$$
\begin{aligned}
\sigma_{t}^{r} f(x) & :=\left(I-\sigma_{t}\right)^{r} f(x) \\
& =\sum_{k=0}^{\infty}(-1)^{k}\left(\begin{array}{l}
r \\
k
\end{array}\right) \frac{1}{(2 t)^{k}} \int_{-t}^{t} \ldots \int_{-t}^{t} f\left(x+u_{1}+\cdots+u_{k}\right) \mathrm{d} u_{1} \ldots \mathrm{d} u_{k}
\end{aligned}
$$

where $\left(\begin{array}{l}r \\ k\end{array}\right)$ are the binomial coefficients.

Since

$$
\left|\left(\begin{array}{l}
\alpha \\
k
\end{array}\right)\right| \leq \frac{c}{k^{\alpha+1}}, \quad k \in \mathbb{N}
$$

(see $[19$, p.14, (1.51)]), we have

$$
\sum_{k=0}^{\infty}\left|\left(\begin{array}{l}
\alpha \\
k
\end{array}\right)\right|<\infty
$$

and therefore

$$
\left\|\sigma_{t}^{\alpha} f\right\|_{p q, \omega} \leq c\|f\|_{p q, \omega}<\infty,
$$

for $f \in L_{\omega}^{p q}(\mathbb{T}), 1<p, q<\infty, \omega \in A_{p}$.

For $1<p, q<\infty, f \in L_{\omega}^{p q}(\mathbb{T})$ and $r \in \mathbb{R}^{+}$, we define the fractional modulus of smoothness of index $r$ as

$$
\Omega_{r}(f, \delta)_{p q, \omega}:=\sup _{0<h_{i}, t<\delta}\left\|\prod_{i=1}^{[r]}\left(I-\sigma_{h_{i}}\right)\left(I-\sigma_{t}\right)^{r-[r]} f\right\|_{p q, \omega^{\prime}}
$$

where $[r]:=\max \{n \in \mathbb{N}: n \leq r\}$. Since the operator $\sigma_{t}$ is bounded in $L_{\omega}^{p q}(\mathbb{T})$, $1<p, q<\infty, \omega \in A_{p}$ we have by (3) that

$$
\Omega_{r}(f, \delta)_{p q, \omega} \leq c\|f\|_{p q, \omega}
$$

where the constant $c>0$ only depends on $r, p, q$ and $C_{A_{p}}$.

Remark 1.1. Let $r \in \mathbb{R}^{+}, 1<p, q<\infty, \omega \in A_{p}$ and $f \in L_{\omega}^{p q}(\mathbb{T})$. For $\delta>0$, the modulus of smoothness $\Omega_{r}(f, \delta)_{p q, \omega}$ has the following properties.

(i) $\Omega_{r}(f, \delta)_{p q, \omega}$ is sub-additive in $f$, and a non-negative, non-decreasing function of $\delta \geq 0$.

(ii) $\lim _{\delta \rightarrow 0} \Omega_{r}(f, \delta)_{p q, \omega}=0$. 
By $E_{n}(f)_{p q, \omega}$ we denote the best approximation of $f \in L_{\omega}^{p q}(\mathbb{T})$ by polynomials in $\mathcal{T}_{n}$, the set of trigonometric polynomials of degree $\leq n$ :

$$
E_{n}(f)_{p q, \omega}=\inf _{T_{n} \in \mathcal{T}_{n}}\left\|f-T_{n}\right\|_{p q, \omega} .
$$

In this paper we will use the following notations:

$$
\begin{aligned}
& A(x) \approx B(x) \Leftrightarrow \exists c_{1}, c_{2}>0: c_{1} B(x) \leq A(x) \leq c_{2} B(x) \\
& A(x) \preceq B(x) \Leftrightarrow \exists c>0: A(x) \leq c B(x) .
\end{aligned}
$$

Our main results are now the following.

Theorem 1.2. If $r \in \mathbb{R}^{+}, 1<p, q<\infty, \omega \in A_{p}$ and $f \in L_{\omega}^{p q}(\mathbb{T})$, then there exists a constant $c>0$ depending only on $r, p, q$ and $C_{A_{p}}$ such that

$$
E_{n}(f)_{p q, \omega} \leq c \Omega_{r}\left(f, \frac{1}{n+1}\right)_{p q, \omega}
$$

holds for $n+1 \in \mathbb{N}$.

The analogues of this direct approximation theorem were obtained in [10] for $r \in \mathbb{N}, f \in L_{\omega}^{p}(\mathbb{T}), 1<p<\infty, \omega \in A_{p}$ and in [2] for $r \in \mathbb{N}, f \in L_{\omega}^{p q}(\mathbb{T})$, $1<p, q<\infty, \omega \in A_{p}$ with the modulus of smoothness

$$
W_{r}\left(f, \frac{1}{n}\right)_{L_{\omega}^{p q}}:=\sup _{0 \leq h_{i} \leq 1 / n}\left\|\prod_{i=1}^{r}\left(I-\sigma_{h_{i}}\right) f\right\|_{L_{\omega}^{p q}},
$$

and in [1] for $r \in \mathbb{R}^{+}, f \in L_{\omega}^{p}(\mathbb{T}), 1<p<\infty, \omega \in A_{p}$ with the fractional modulus of smoothness (4).

For $f \in L_{\omega}^{p q}(\mathbb{T}), t, r>0$ and $1<p, q<\infty$, the Peetre $K$-functional is defined as

$$
K_{r}\left(f, t ; L_{\omega}^{p q}, W_{p q, \omega}^{r}\right):=\inf _{g \in W_{p q, w}^{r}}\left\{\|f-g\|_{L_{\omega}^{p q}}+t^{r}\left\|g^{(r)}\right\|_{L_{\omega}^{p q}}\right\} .
$$

Here $W_{p q, \omega}^{r}:=\left\{g(x) \in L_{\omega}^{p q}: g^{(r)} \in L_{\omega}^{p q}\right\}$.

We define the realization functional for $f \in L_{\omega}^{p q}(\mathbb{T})$ by

$$
R_{r}\left(f, 1 / n, L_{\omega}^{p q}\right):=\left\{\left\|f-t_{n}^{*}\right\|_{L_{\omega}^{p q}}+\frac{1}{n^{r}}\left\|\left(t_{n}^{*}\right)^{(r)}\right\|_{L_{\omega}^{p q}}\right\}
$$

for $r>0,1<p, q<\infty, n \in \mathbb{N}$. Here $t_{n}^{*}$ denotes the best approximating trigonometric polynomial for $f$. The following theorem holds.

Theorem 1.3. If $\mathbb{R}^{+}, f \in L_{\omega}^{p q}(\mathbb{T}), 1<p, q<\infty$ and $\omega \in A_{p}$, then the equivalence

$$
\Omega_{r}\left(f, \frac{1}{n}\right)_{p q, \omega} \approx R_{2 r}\left(f, 1 / n, L_{w}^{p q}\right)
$$

holds for $n=1,2,3, \ldots$. Furthermore, we have, for $\delta \geq 0$,

$$
\Omega_{r}(f, \delta)_{p q, \omega} \approx K_{2 r}\left(f, \delta ; L_{w}^{p q}, W_{p q, w}^{r}\right) .
$$

Here the equivalence constants only depend on $r, p, q$ and $C_{A_{p}}$. 
Corollary 1.4. Let $r \in \mathbb{R}^{+}, f \in L_{w}^{p q}(\mathbb{T}), 1<p, q<\infty$, and $\omega \in A_{p}$. Then

$$
\Omega_{r}(f, \lambda \delta)_{p q, \omega} \preceq(1+[\lambda])^{2 r} \Omega_{r}(f, \delta)_{p q, \omega}, \quad \delta, \lambda>0
$$

and

$$
\Omega_{r}(f, \delta)_{p q, \omega} \delta^{-2 r} \preceq \Omega_{r}\left(f, \delta_{1}\right)_{p q, \omega} \delta_{1}^{-2 r}, \quad 0<\delta_{1} \leq \delta .
$$

An improvement of (5) is given by the following theorem.

Theorem 1.5. If $r \in \mathbb{R}^{+}, f \in L_{\omega}^{p q}(\mathbb{T}), 1<p, q<\infty$ and $\omega \in A_{p}$, then there exists $a$ constant $c>0$ depending on $r, p, q$ and $C_{A_{p}}$ such that for $n=1,2,3, \ldots$

$$
\left(\prod_{j=1}^{n} E_{j}(f)_{p q, \omega}\right)^{1 / n} \leq c \Omega_{r}\left(f, \frac{1}{n}\right)_{p q, \omega}
$$

Remark 1.6. The inequality (8) is never worse than the classical Jackson inequality. Since $E_{n}(f)_{p q, \omega} \rightarrow 0$ as $n \rightarrow \infty$ we obtain that

$$
E_{n}(f)_{p q, \omega} \leq\left(\prod_{j=1}^{n} E_{j}(f)_{p q, \omega}\right)^{1 / n} \leq c \Omega_{r}\left(f, \frac{1}{n}\right)_{p q, \omega} .
$$

On the other hand, in some cases the inequality (8) gives better results than the classical Jackson inequality. For example, if $E_{n}(f)_{p q, \omega}=2^{-n}$, then the classical Jackson inequality implies $\Omega_{r}\left(f, \frac{1}{n}\right)_{p q, \omega} \geq c 2^{-n}$ but inequality (8) yields $\Omega_{r}\left(f, \frac{1}{n}\right)_{p q, \omega} \geq c 2^{-n / 2}$.

An analogue of Theorem 1.5 for the space $L^{\infty}$ was proved in [18]. In [2], the weak converse of (5)

$$
\Omega_{r}\left(f, \frac{1}{n}\right)_{L_{\omega}^{p q}} \leq \frac{c}{n^{2 r}} \sum_{v=0}^{n}(v+1)^{2 r-1} E_{v}(f)_{L_{\omega}^{p q}}^{p,}
$$

for $r \in \mathbb{N}, f \in L_{\omega}^{p q}(\mathbb{T}), \omega \in A_{p}$ and $1<p, q<\infty$ was obtained.

Theorem 1.7. Let $1<p<\infty$ and $1<q \leq 2$ or $p>2$ and $q \geq 2, \omega \in A_{p}$, $f \in L_{\omega}^{p q}(\mathbb{T})$. If $n \in \mathbb{N}, r \in \mathbb{R}^{+}$and $\gamma:=\min \{2, q\}$, then there is a constant $c>0$ only depending on $r, q, p$ and $C_{A_{p}}$ such that

$$
\Omega_{r}\left(f, \frac{1}{n}\right)_{p q, \omega} \leq \frac{c}{n^{2 r}}\left(\sum_{v=1}^{n} v^{2 \gamma r-1} E_{v-1}^{\gamma}(f)_{p q, \omega}\right)^{1 / \gamma} .
$$

The analogues of this improved converse theorem were proven in [15] for $r \in \mathbb{N}$, $f \in L_{\omega}^{p}(\mathbb{T}), 1<p<\infty, \omega \in A_{p}$ with the modulus of smoothness $W_{r}\left(f, \frac{1}{n}\right)_{L_{\omega}^{p}}$; in [1] for $r \in \mathbb{R}^{+}, f \in L_{\omega}^{p}(\mathbb{T}), 1<p<\infty, \omega \in A_{p}$ with the fractional modulus of smoothness (4); in [14] for $r \in \mathbb{N}, f \in L_{\omega}^{p q}(\mathbb{T}), 1<p<\infty$ and $1<q \leq 2$ or $p>2$ and $q \geq 2, \omega \in A_{p}$ with $W_{r}\left(f, \frac{1}{n}\right)_{L_{\omega}^{p q}} ;$ in [20] for $r \in \mathbb{R}^{+}, f \in L_{\omega}^{p q}(\mathbb{T}), 1<p<\infty$ 
and $1<q \leq 2$ or $p>2$ and $q \geq 2, \omega \in A_{p}$ with a modulus of smoothness defined by Ky [16].

The inequality (10) is better than (9). Indeed, using the fact that $x^{\gamma}$ is convex for $\gamma=\min \{2, p\}$ we obtain that

$$
\begin{aligned}
& \left(v v^{2 r-1} E_{v}(f)_{p q, \omega}\right)^{\gamma}-\left((v-1) v^{2 r-1} E_{v}(f)_{p q, \omega}\right)^{\gamma} \\
& \quad \leq\left(\sum_{\mu=1}^{v} \mu^{2 r-1} E_{\mu}(f)_{p q, \omega}\right)^{\gamma}-\left(\sum_{\mu=1}^{v-1} \mu^{2 r-1} E_{\mu}(f)_{p q, \omega}\right)^{\gamma} .
\end{aligned}
$$

Taking the summation over $v$, we obtain that

$$
\begin{aligned}
& \sum_{\nu=1}^{n}\left\{\left(v v^{2 r-1} E_{\nu}(f)_{p q, \omega}\right)^{\gamma}-\left((v-1) v^{2 r-1} E_{v}(f)_{p q, \omega}\right)^{\gamma}\right\} \\
& \left.\leq \sum_{\nu=1}^{n}\left\{\left(\sum_{\mu=1}^{v} \mu^{2 r-1} E_{\mu}(f)_{p q, \omega}\right)\right)^{\gamma}-\left(\sum_{\mu=1}^{v-1} \mu^{2 r-1} E_{\mu}(f)_{p q, \omega}\right)^{\gamma}\right\},
\end{aligned}
$$

hence we have the inequality

$$
\left\{\sum_{\nu=1}^{n} v^{2 \gamma r-1} E_{v-1}^{\gamma}(f)_{p q, \omega}\right\}^{1 / \gamma} \leq 2 \sum_{\nu=1}^{n} v^{2 r-1} E_{v-1}(f)_{p q, \omega} .
$$

We give the Marcinkiewicz multiplier and Littlewood-Paley theorems in $L_{\omega}^{p q}(\mathbb{T})$ which are used in the proofs of previous Theorems.

Theorem 1.8. Let $\lambda_{0}, \lambda_{1}, \cdots$ be a sequence of real numbers such that

$$
\left|\lambda_{l}\right| \leq M \text { and } \sum_{v=2^{l-1}}^{2^{l}-1}\left|\lambda_{v}-\lambda_{v+1}\right| \leq M
$$

for all $v, l \in \mathbb{N}$. If $1<p, q<\infty, \omega \in A_{p}$ and $f \in L_{\omega}^{p q}(\mathbb{T})$ with Fourier series $\sum_{v=0}^{\infty}\left(a_{v}(f) \cos v x+b_{v}(f) \sin v x\right)$, then there exists $h \in L_{\omega}^{p q}(\mathbb{T})$ such that the series $\sum_{v=0}^{\infty} \lambda_{v}\left(a_{v}(f) \cos v x+b_{v}(f) \sin v x\right)$ is the Fourier series of $h$ and

$$
\|h\|_{p q, \omega} \leq C\|f\|_{p q, \omega},
$$

where $C$ does not depend on $f$.

Theorem 1.9. Let $v \in \mathbb{N}, 1<p, q<\infty, \omega \in A_{p}$ and $f \in L_{\omega}^{p q}(\mathbb{T})$ with Fourier series $\sum_{v=0}^{\infty}\left(a_{v}(f) \cos v x+b_{v}(f) \sin v x\right)$, then there exist constants $c_{1}$ and $c_{2}$ independent of $f$ such that

$$
c_{1}\left\|\left(\sum_{\mu=v}^{\infty}\left|\Delta_{\mu}\right|^{2}\right)^{1 / 2}\right\|_{p q, \omega} \leq\|f\|_{p q, \omega} \leq c_{2}\left\|\left(\sum_{\mu=v}^{\infty}\left|\Delta_{\mu}\right|^{2}\right)^{1 / 2}\right\|_{p q, \omega},
$$

where

$$
\Delta_{\mu}:=\Delta_{\mu}(x, f):=\sum_{\nu=2^{\mu-1}}^{2^{\mu}-1}\left(a_{\nu}(f) \cos v x+b_{\nu}(f) \sin v x\right) .
$$




\section{Proof of the main results}

From $[5,14]$ we recall four important properties of the spaces $L_{\omega}^{p q}(\mathbb{T})$.

Lemma A ([5] or [13, prop. 5.1.2]) For $1<p, q<\infty$, there exists a $c>0$ such that for every $f \in L_{\omega}^{p q}(\mathbb{T})$

$$
c^{-1}\|f\|_{p q, \omega} \leq \sup \left|\int_{\mathbb{T}} f(x) g(x) w(x) \mathrm{d} x\right| \leq c\|f\|_{p q, \omega},
$$

where the supremum is taken over all functions $g$ for which $\|g\|_{p^{\prime} q^{\prime}, \omega} \leq 1$.

Lemma B [14]. Let $1<p<\infty$ and $1<q \leq 2$. Then for an arbitrary system of functions $\left\{\varphi_{j}(x)\right\}_{j=1}^{m}, \varphi_{j} \in L_{\omega}^{p q}(\mathbb{T})$ we have

$$
\left\|\left(\sum_{j=1}^{m} \varphi_{j}^{2}\right)^{1 / 2}\right\|_{p q, \omega} \leq c\left(\sum_{j=1}^{m}\left\|\varphi_{j}\right\|_{p q, \omega}^{q}\right)^{1 / q}
$$

with a constant $c$ independent of $\varphi_{j}$ and $m$.

Lemma C [14]. Let $2<p<\infty$ and $q \geq 2$. For an arbitrary system $\left\{\varphi_{j}(x)\right\}_{j=1}^{m}$, $\varphi_{j} \in L_{\omega}^{p q}(\mathbb{T})$, we have

$$
\left\|\left(\sum_{j=1}^{m} \varphi_{j}^{2}\right)^{1 / 2}\right\|_{p q, \omega} \leq c\left(\sum_{j=1}^{m}\left\|\varphi_{j}\right\|_{p q, \omega}^{2}\right)^{1 / 2}
$$

with a constant $c$ independent of $\varphi_{j}$ and $m$.

Lemma D [14]. Let $1<p, q<\infty, f \in L_{\omega}^{p q}(\mathbb{T})$ and $w \in A_{p}$. If $B_{k, \mu}(x)=$ $a_{k}(f) \cos \left(k+\mu \frac{\pi}{2}\right) x+b_{k}(f) \sin \left(k+\mu \frac{\pi}{2}\right) x$, where $a_{k}, b_{k}$ are the Fourier coefficients of $f$, then

$$
\left\|\sum_{k=2^{i}+1}^{2^{i+1}} k^{\mu} B_{k, \mu}\right\|_{p q, \omega} \leq c 2^{i \mu} E_{2^{i}}(f)_{p q, \omega},
$$

where the constant $c$ is independent of $f$ and $i$.

Proof of Theorem 1.8. We define a linear operator

$$
\mathrm{T} f(x):=\sum_{v=0}^{\infty} \lambda_{v}\left(a_{v}(f) \cos v x+b_{v}(f) \sin v x\right)
$$

for $f \in L_{\omega}^{p q}(\mathbb{T})$ which is bounded (in particular is of weak type $(p, p)$ ) in $L_{\omega}^{p}(\mathbb{T})$ for every $p>1$ by [4, Th. 4.4]. Therefore the hypothesis of the interpolation theorem for Lorentz spaces [3, Th. 4.13] is fulfilled. Applying this theorem we get the desired result (11).

Proof of Theorem 1.9. Let us define a quasilinear operator

$$
T f(x):=\left(\sum_{\mu=v}^{\infty}\left|\Delta_{\mu}(x, f)\right|^{2}\right)^{1 / 2} .
$$


This operator is bounded in $L_{\omega}^{p}(\mathbb{T})$ for every $p>1$ by [4, Th. 4.5]. Therefore the left hand side of the required result (12) is derived by means of the interpolation theorem for Lorentz spaces [3, Th. 4.13].

Using Hölder's inequality for $f \in L_{\omega}^{p q}(\mathbb{T}) \cap L_{\omega}^{2}(\mathbb{T}), g \in L_{\omega}^{p^{\prime} q^{\prime}}(\mathbb{T}) \cap L_{\omega}^{2}(\mathbb{T})$ and the left hand side of (12) we obtain

$$
\begin{aligned}
\int_{\mathbb{T}}|f(x) g(x)| \omega(x) \mathrm{d} x=\int_{\mathbb{T}}\left|\sum_{\mu=1}^{\infty} \Delta_{\mu}(x, f) \Delta_{\mu}(x, g)\right| \omega(x) \mathrm{d} x \\
\leq \int_{\mathbb{T}} \sum_{\mu=1}^{\infty}\left|\Delta_{\mu}(x, f) \Delta_{\mu}(x, g)\right| \omega(x) \mathrm{d} x \\
\leq \int_{\mathbb{T}}\left[\sum_{\mu=1}^{\infty}\left|\Delta_{\mu}(x, f)\right|^{2}\right]^{1 / 2}\left[\sum_{\mu=1}^{\infty}\left|\Delta_{\mu}(x, g)\right|^{2}\right]^{1 / 2} \omega(x) \mathrm{d} x \\
\leq\left\|\left[\sum_{\mu=1}^{\infty}\left|\Delta_{\mu}(x, f)\right|^{2}\right]^{1 / 2}\right\|\left\|_{p q, \omega}\right\|\left[\sum_{\mu=1}^{\infty}\left|\Delta_{\mu}(x, g)\right|^{2}\right]^{1 / 2} \|_{p^{\prime} q^{\prime}, \omega} \\
\leq C\left\|\left[\sum_{\mu=1}^{\infty}\left|\Delta_{\mu}(x, f)\right|^{2}\right]^{1 / 2}\right\|\left\|_{p q, \omega}\right\| g \|_{p^{\prime} q^{\prime}, \omega} .
\end{aligned}
$$

where $p^{\prime}=p /(p-1), q^{\prime}=q /(q-1)$. Taking the supremum in the last inequality over all functions $g \in L_{\omega}^{p^{\prime} q^{\prime}}(\mathbb{T})$ satisfying $\|g\|_{p^{\prime} q^{\prime}, \omega} \leq 1$, we find, applying Lemma A that

$$
\|f\|_{p q, \omega} \leq C\left\|\left(\sum_{\mu=1}^{\infty}\left|\Delta_{\mu}\right|^{2}\right)^{1 / 2}\right\|_{p q, \omega} .
$$

The density of $L_{\omega}^{p q}(\mathbb{T}) \cap L_{\omega}^{2}(\mathbb{T})$ in $L_{\omega}^{p q}(\mathbb{T})$ yields the last inequality for any $f \in L_{\omega}^{p q}(\mathbb{T})$.

Lemma 2.1. If $0<\alpha \leq \beta, \omega \in A_{p}, 1<p, q<\infty$ and $f \in L_{\omega}^{p q}(\mathbb{T})$ then

$$
\Omega_{\beta}(f, \cdot)_{p q, \omega} \leq c \Omega_{\alpha}(f, \cdot)_{p q, \omega} .
$$

Proof. The proof of Lemma 2.1 is similar to the proof of [1, Lemma 1].

Lemma 2.2. Let $r \in \mathbb{R}^{+}, 1<p, q<\infty, \omega \in A_{p}$ and $T_{n} \in \mathcal{T}_{n}$ for $n=1,2, \cdots$. Then

$$
\Omega_{r}\left(T_{n}, \frac{1}{n}\right)_{p q, \omega} \preceq \frac{1}{n^{2 r}}\left\|T_{n}^{(2 r)}\right\|_{p q, \omega}
$$

holds with some constant only depending on $r, p, q$ and $C_{A_{p}}$.

Proof. For all $x \geq 0$, we have that

$$
\left(1-\frac{\sin x}{x}\right)_{*} \leq x^{2}
$$

where

$$
\left(1-\frac{\sin x}{x}\right)_{*}:= \begin{cases}1-\frac{\sin x}{x} & \text { if } x \geq 0 \\ 0 & \text { if } x=0\end{cases}
$$


For $0<t$ and $h_{i} \leq \frac{1}{n}$, we have that

$$
\begin{aligned}
& \left\|\prod_{i=1}^{[r]}\left(I-\sigma_{h_{i}}\right)\left(I-\sigma_{t}\right)^{r-[r]} T_{n}\right\|_{p q, \omega} \\
& =\| \sum_{v=0}^{n}\left(1-\frac{\sin v h_{1}}{v h_{1}}\right)_{*} \ldots\left(1-\frac{\sin v h_{[r]}}{v h_{[r]}}\right)_{*}\left(1-\frac{\sin v t}{v t}\right)_{*}^{r-[r]} \\
& A_{v}\left(T_{n}, x\right) \|_{p q, \omega} \\
& =\| \sum_{v=1}^{n} \frac{\left(1-\frac{\sin v h_{1}}{v h_{1}}\right)\left(v h_{1}\right)^{2}}{\left(v h_{1}\right)^{2}} \cdots \frac{\left(1-\frac{\sin v h_{[r]}}{v h_{[r]}}\right)\left(v h_{[r]}\right)^{2}}{\left(v h_{[r]}\right)^{2}}\left(\frac{1-\frac{\sin v t}{v t}}{(v t)^{2}}\right)^{r-[r]} \\
& (v t)^{2(r-[r])} A_{v}\left(T_{n}, x\right) \|_{p q, \omega} \\
& \leq n^{-2 r} \| \sum_{v=1}^{n} \frac{\left(1-\frac{\sin v h_{1}}{v h_{1}}\right)}{\left(v h_{1}\right)^{2}} v^{2} \ldots \frac{\left(1-\frac{\sin v h_{[r]}}{v h_{[r]}}\right)}{\left(v h_{[r]}\right)^{2}} v^{2}\left(\frac{1-\frac{\sin v t}{v t}}{(v t)^{2}}\right)^{r-[r]} \\
& v^{2(r-[r])} A_{v}\left(T_{n}, x\right) \|_{p q, \omega} \\
& \leq n^{-2 r}\left\|\sum_{\nu=1}^{n} v^{2 r} \frac{\left(1-\frac{\sin v h_{1}}{v h_{1}}\right)}{\left(v h_{1}\right)^{2}} \cdots \frac{\left(1-\frac{\sin v h_{[r]}}{v h_{[r]}}\right)}{\left(v h_{[r]}\right)^{2}}\left(\frac{1-\frac{\sin v t}{v t}}{(v t)^{2}}\right)^{r-[r]} A_{v}\left(T_{n}, x\right)\right\|_{p q, \omega} \text {. }
\end{aligned}
$$

Applying Theorem 1.8 we obtain that

$$
\left\|\prod_{i=1}^{[r]}\left(I-\sigma_{h_{i}}\right)\left(I-\sigma_{t}\right)^{r-[r]} T_{n}\right\|_{p q, \omega} \preceq n^{-2 r}\left\|\sum_{\nu=1}^{n} v^{2 r} A_{\nu}\left(T_{n}, x\right)\right\|_{p q, \omega} .
$$

For $v=1,2,3, \ldots$ we have

$$
A_{v}\left(T_{n}, x\right)=A_{v}\left(T_{n}, x+\frac{r \pi}{v}\right) \cos r \pi+A_{v}\left(\tilde{T}_{n}, x+\frac{r \pi}{v}\right) \sin r \pi,
$$

where $\tilde{T}_{n}$ is the Fourier conjugate of $T_{n}$. Therefore

$$
\begin{aligned}
& \left\|\prod_{i=1}^{[r]}\left(I-\sigma_{h_{i}}\right)\left(I-\sigma_{t}\right)^{r-[r]} T_{n}\right\|_{p q, \omega} \\
& \preceq n^{-2 r}\left\|\sum_{v=1}^{n} v^{2 r}\left(A_{v}\left(T_{n}, x+\frac{r \pi}{v}\right) \cos r \pi+A_{v}\left(\tilde{T}_{n}, x+\frac{r \pi}{v}\right) \sin r \pi\right)\right\|_{p q, \omega} \\
& \left.\preceq n^{-2 r}\left(\left\|\sum_{v=1}^{n} v^{2 r} A_{v}\left(T_{n}, x+\frac{r \pi}{v}\right)\right\|_{p q, \omega}+\| \sum_{v=1}^{n} v^{2 r} A_{v}\left(\tilde{T}_{n}, x+\frac{r \pi}{v}\right)\right) \|_{p q, \omega}\right) .
\end{aligned}
$$

Since

$$
A_{v}\left(T_{n}^{(2 r)}, x\right)=v^{2 r} A_{v}\left(T_{n}, x+\frac{r \pi}{v}\right)
$$


for $v=1,2,3, \cdots$, we find

$$
\begin{aligned}
& \Omega_{r}\left(T_{n}, \frac{1}{n}\right)_{p q, \omega} \\
& \quad \preceq n^{-2 r}\left(\left\|\sum_{v=1}^{n} v^{2 r} A_{v}\left(T_{n}, x+\frac{r \pi}{v}\right)\right\|_{p q, \omega}+\left\|\sum_{v=1}^{n} v^{2 r} A_{v}\left(\tilde{T}_{n}, x+\frac{r \pi}{v}\right)\right\|_{p q, \omega}\right) \\
& \quad \preceq n^{-2 r}\left(\left\|T_{n}^{(2 r)}\right\|_{p q, \omega}+\left\|\tilde{T}_{n}(2 r)\right\|_{p q, \omega}\right) \preceq n^{-2 r}\left\|T_{n}(2 r)\right\|_{p q, \omega} \cdot
\end{aligned}
$$

Lemma 2.3. Let $r \in \mathbb{R}_{+}, 1<p, q<\infty, \omega \in A_{p}$ and $T_{n} \in \mathcal{T}_{n}$. For $n=1,2, \cdots$, we have that

$$
\frac{1}{n^{2 r}}\left\|T_{n}^{(2 r)}\right\|_{p q, \omega} \preceq \Omega_{r}\left(T_{n}, \frac{1}{n}\right)_{p q, \omega}
$$

with some constant depending only on $r, p, q$ and $C_{A_{p}}$.

Proof.

$$
\begin{gathered}
n^{-2 r}\left\|T_{n}^{(2 r)}\right\|_{p q, \omega}=n^{-2 r}\left\|\sum_{v=1}^{n} v^{2 r} A_{v}\left(T_{n}, x+\frac{r \pi}{v}\right)\right\|_{p q, \omega} \\
=n^{-2 r}\left\|\sum_{v=1}^{n} v^{2 r}\left(A_{v}\left(T_{n}, x\right) \cos r \pi+A_{v}\left(\tilde{T}_{n}, x\right) \sin r \pi\right)\right\|_{p q, \omega} \\
\leq n^{-2 r}\left\|\sum_{v=1}^{n} v^{2 r} A_{v}\left(T_{n}, x\right) \cos r \pi\right\|_{p q, \omega} \\
\quad+n^{-2 r}\left\|\sum_{v=1}^{n} v^{2 r} A_{v}\left(\tilde{T}_{n}, x\right) \sin r \pi\right\|_{p q, \omega} \\
=\| \sum_{v=1}^{n} \cos r \pi\left(\frac{\left(\frac{v}{n}\right)^{2}}{\left.1-\frac{\sin \frac{v}{n}}{\frac{v}{n}}\right)^{r}\left(1-\frac{\sin \frac{v}{n}}{\frac{v}{n}}\right)^{r} A_{v}\left(T_{n}, x\right) \|_{p q, \omega}}\right. \\
\quad+\left\|\sum_{v=1}^{n} \sin r \pi\left(\frac{\left(\frac{v}{n}\right)^{2}}{1-\frac{\sin \frac{v}{n}}{\frac{v}{n}}}\right)^{r}\left(1-\frac{\sin \frac{v}{n}}{\frac{v}{n}}\right)^{r} A_{v}\left(\tilde{T}_{n}, x\right)\right\|_{p q, \omega} .
\end{gathered}
$$

Applying Theorem 1.8 and the linearity of the conjugate operator we get

$$
\begin{aligned}
n^{-2 r}\left\|T_{n}^{(2 r)}\right\|_{p q, \omega} \preceq \| & \sum_{v=1}^{n}\left(1-\frac{\sin \frac{v}{n}}{\frac{v}{n}}\right)^{r} A_{v}\left(T_{n}, x\right) \|_{p q, \omega} \\
& +\left\|\sum_{v=1}^{n}\left(1-\frac{\sin \frac{v}{n}}{\frac{v}{n}}\right)^{r} A_{v}\left(\tilde{T}_{n}, x\right)\right\|_{p q, \omega} \\
=\| & \left\|\sum_{v=1}^{n}\left(1-\frac{\sin \frac{v}{n}}{\frac{v}{n}}\right)^{r} A_{v}\left(T_{n}, x\right)\right\|_{p q, \omega} \\
& +\left\|\left(\sum_{v=1}^{n}\left(1-\frac{\sin \frac{v}{n}}{\frac{v}{n}}\right)^{r} A_{v}\left(T_{n}, x\right)\right)^{\sim}\right\|_{p q, \omega}
\end{aligned}
$$


From the boundedness of the conjugate operator [14] we have

$$
\begin{aligned}
& n^{-2 r}\left\|T_{n}^{(2 r)}\right\|_{p q, \omega} \\
& \preceq\left\|\sum_{v=1}^{n}\left(1-\frac{\sin \frac{v}{n}}{\frac{v}{n}}\right)^{r} A_{v}\left(T_{n}, x\right)\right\|_{p q, \omega}+\left\|\sum_{v=1}^{n}\left(1-\frac{\sin \frac{v}{n}}{\frac{v}{n}}\right)^{r} A_{v}\left(T_{n}, x\right)\right\|_{p q, \omega} \\
& \preceq\left\|\left(I-\sigma_{\frac{1}{n}}\right)^{r} T_{n}\right\|_{p q, \omega} \preceq \sup _{0<h_{i}, u<1 / n}\left\|\prod_{i=1}^{[r]}\left(I-\sigma_{h_{i}}\right)\left(I-\sigma_{u}\right)^{r-[r]} T_{n}\right\|_{p q, \omega} \\
& \preceq \Omega_{r}\left(T_{n}, \frac{1}{n}\right)_{p q, \omega} .
\end{aligned}
$$

Proof of Theorem 1.2. From Lemma 2.1 and [2, Th. 1.1] we have

$$
E_{n}(f)_{p q, \omega} \leq c \Omega_{[r]+1}\left(f, \frac{1}{n+1}\right)_{p q, \omega} \leq C \Omega_{r}\left(f, \frac{1}{n+1}\right)_{p q, \omega}
$$

for $n+1 \in \mathbb{N}$ and the assertion (5) follows.

Lemma 2.4. Let $1<p, q<\infty, \omega \in A_{p}, f \in L_{\omega}^{p q}(\mathbb{T})$ and $\gamma>0$. Then for any $0<t<2$,

$$
\Omega_{\gamma}(f, t)_{p q, \omega} \preceq t^{\gamma}\left\|f^{(\gamma)}\right\|_{p q, \omega} .
$$

Proof. There is some $n=1,2,3, \ldots$ such that $(1 / n)<t \leq(2 / n)$. From Lemma 2.2 we get

$$
\Omega_{\gamma}(f, t)_{p q, \omega} \leq \Omega_{\gamma}\left(f-T_{n}, t\right)_{p q, \omega}+\Omega_{\gamma}\left(T_{n}, t\right)_{p q, \omega} \preceq E_{n}(f)_{p q, \omega}+t^{2 \gamma}\left\|T_{n}^{(2 \gamma)}\right\|_{p q, \omega} .
$$

On the other hand applying [20, (3.9) and Th. 1.3] and Theorem 1.2 we have

$$
E_{n}(f)_{p q, \omega} \preceq \frac{1}{n^{2 \gamma}} E_{n}\left(f^{(2 \gamma)}\right)_{p q, \omega} \preceq \frac{1}{n^{2 \gamma}} \Omega_{\gamma}\left(f^{(2 \gamma)}, \frac{1}{n}\right)_{p q, \omega} \preceq t^{2 \gamma}\left\|f^{(2 \gamma)}\right\|_{p q, \omega} .
$$

Using Theorem 1.2 and [20, Th. 1.3] the proof is completed.

Proof of Theorem 1.3. We have to show that (6) holds. Let $T_{n}$ be the near best approximating trigonometric polynomial to $f$. From Theorem 1.2

$$
\left\|f-T_{n}\right\|_{p q, \omega} \preceq E_{n}(f)_{p q, \omega} \leq c \Omega_{r}\left(f, \frac{1}{n+1}\right)_{p q, \omega} .
$$

Applying Lemma 2.3, we find that

$$
\begin{aligned}
& \frac{1}{n^{2 r}}\left\|^{(2 r}{ }^{(2 r}\right\|_{p q, \omega} \preceq \Omega_{r}\left(T_{n}, \frac{1}{n}\right)_{p q, \omega} \preceq \Omega_{r}\left(T_{n}-f, \frac{1}{n}\right)_{p q, \omega}+\Omega_{r}\left(f, \frac{1}{n}\right)_{p q, \omega} \\
& \preceq\left\|f-T_{n}\right\|_{p q, \omega}+\Omega_{r}\left(f, \frac{1}{n}\right)_{p q, \omega} \preceq \Omega_{r}\left(f, \frac{1}{n}\right)_{p q, \omega}
\end{aligned}
$$


and

$$
\left\|f-T_{n}\right\|_{p q, \omega}+\frac{1}{n^{2 r}}\left\|T_{n}^{(2 r)}\right\|_{p q, \omega} \preceq \Omega_{r}\left(f, \frac{1}{n}\right)_{p q, \omega} .
$$

On the other hand using Lemma 2.2

$$
\begin{aligned}
\Omega_{r}\left(f, \frac{1}{n}\right)_{p q, \omega} & \leq \Omega_{r}\left(f-T_{n}, \frac{1}{n}\right)_{p q, \omega}+\Omega_{r}\left(T_{n}, \frac{1}{n}\right)_{p q, \omega} \\
& \preceq\left\|f-T_{n}\right\|_{p q, \omega}+\frac{1}{n^{2 r}}\left\|T_{n}^{(2 r)}\right\|_{p q, \omega}=R_{2 r}\left(f, \frac{1}{n}, L_{\omega}^{p q}\right) .
\end{aligned}
$$

This completes the proof of (6). Using Lemma 2.4, properties of the modulus of smoothness and of the $K$-functional ( 7 ) are proven.

Proof of Theorem 1.5. By Corollary 1.4 we have for $v \leq n$

$$
\Omega_{r}(f, 1 / v)_{p q, \omega} \leq(1+n / v)^{2 r} \Omega_{r}(f, 1 / n)_{p q, \omega}
$$

and

$$
\prod_{v=1}^{n} \Omega_{r}(f, 1 / v)_{p q, \omega} \leq \prod_{v=1}^{n}(1+n / v)^{2 r}\left(\Omega_{r}(f, 1 / n)_{p q, \omega}\right)^{n} .
$$

For every $n$ we have

$$
\prod_{v=1}^{n}(1+n / v)^{2 r} \leq\left(\frac{2 n}{\sqrt[n]{n !}}\right)^{2 r}
$$

Using Stirling's formula

$$
n ! \asymp \sqrt{2 \pi n} n^{n} e^{-n} e^{\theta(n)} \text { with }|\theta(n)| \leq 1 /(12 n)
$$

we get

$$
\prod_{v=1}^{n}(1+n / v)^{2 r} \leq 2^{2 r} e^{4 r}
$$

Thus

$$
\left(\prod_{v=1}^{n} \Omega_{r}(f, 1 / v)_{p q, \omega}\right)^{1 / n} \leq c \Omega_{r}(f, 1 / n)_{p q, \omega} .
$$

From (5) and the property $E_{n}(f)_{p q, \omega} \rightarrow 0$ as $n \rightarrow \infty$ we find

$$
\left(\prod_{v=1}^{n} E_{v}(f)_{p q, \omega}\right)^{1 / n} \leq\left(\prod_{v=1}^{n} \Omega_{r}(f, 1 / v)_{p q, \omega}\right)^{1 / n} \leq c \Omega_{r}(f, 1 / n)_{p q, \omega} .
$$

Proof of Theorem 1.7. For $1<p, q<\infty, \omega \in A_{p}$, let $f \in L_{\omega}^{p q}(\mathbb{T})$ be such that $\int_{0}^{2 \pi} f(x) \mathrm{d} x=0$. We assume that $f$ has Fourier series (1). We choose $m \in \mathbb{N}$ such that $2^{m} \leq n<2^{m+1}$. Let us denote $S_{n}(x):=S_{n}(x, f):=\sum_{k=0}^{n} A_{k}(f, x)$, for $x \in \mathbb{T}$, where $A_{k}(f, x)=a_{k}(f) \cos k x+b_{k}(f) \sin k x$. By [14, Prop. 3.4], we have that

$$
\left\|f-S_{n}\right\|_{p q, \omega} \leq c E_{n}(f)_{p q, \omega}
$$


It is well-known that $\sigma_{t, h_{1}, h_{2}, \ldots, h_{[r]}} f:=\prod_{i=1}^{[r]}\left(I-\sigma_{h_{i}}\right)\left(I-\sigma_{t}\right)^{r-[r]} f$ has Fourier series

$$
\begin{aligned}
& \sigma_{t, h_{1}, h_{2}, \ldots, h_{[r]}}^{r}(\cdot) \\
& \quad \sim \sum_{v=0}^{\infty}\left(1-\frac{\sin v t}{v t}\right)_{*}^{r-[r]}\left(1-\frac{\sin v h_{1}}{v h_{1}}\right)_{*} \ldots\left(1-\frac{\sin v h_{[r]}}{v h_{[r]}}\right)_{*} A_{v}(f, x) .
\end{aligned}
$$

Moreover

$$
\begin{aligned}
& \sigma_{t, h_{1}, h_{2}, \ldots, h_{[r]}}^{r} f(\cdot) \\
& \quad=\sigma_{t, h_{1}, h_{2}, \ldots, h_{[r]}}^{r}\left(f(\cdot)-S_{2^{m-1}}(\cdot, f)\right)+\sigma_{t, h_{1}, h_{2}, \ldots, h_{[r]}}^{r} S_{2^{m-1}}(\cdot, f) .
\end{aligned}
$$

From (14) and $E_{n}(f)_{p, \omega} \rightarrow 0$ we have

$$
\begin{aligned}
& \left\|\sigma_{t, h_{1}, h_{2}, \ldots, h_{[r]}}^{r}\left(f(\cdot)-S_{2^{m-1}}(\cdot, f)\right)\right\|_{p q, \omega} \\
& \quad \leq c\left\|f(\cdot)-S_{2^{m-1}}(\cdot, f)\right\|_{p q, \omega} \leq c E_{2^{m-1}}(f)_{p q, \omega} \\
& \quad \leq \frac{c}{n^{2 r}}\left\{\sum_{v=1}^{n} v^{2 \gamma r-1} E_{v-1}^{\gamma}(f)_{p, \omega}\right\}^{1 / \gamma} .
\end{aligned}
$$

On the other hand, it follows from (12) that

$$
\left\|\sigma_{t, h_{1}, h_{2}, \ldots, h_{[r]}} S_{2^{m-1}}(\cdot, f)\right\|_{p q, \omega} \leq c\left\|\left\{\sum_{\mu=1}^{m}\left|\delta_{\mu}\right|^{2}\right\}^{1 / 2}\right\|_{p q, \omega}
$$

where

$$
\delta_{\mu}:=\sum_{v=2^{\mu-1}}^{2^{\mu}-1}\left(1-\frac{\sin v t}{v t}\right)^{r-[r]}\left(1-\frac{\sin v h_{1}}{v h_{1}}\right) \ldots\left(1-\frac{\sin v h_{[r]}}{v h_{[r]}}\right) A_{v}(f, x) .
$$

By Lemmas B and C

$$
\left\|\left\{\sum_{\mu=1}^{m}\left|\delta_{\mu}\right|^{2}\right\}^{1 / 2}\right\|_{p q, \omega} \leq\left\{\sum_{\mu=1}^{m}\left\|\delta_{\mu}\right\|_{p q, \omega}^{\gamma}\right\}^{1 / \gamma} .
$$

By Abel's transformation we obtain

$$
\begin{gathered}
\left\|\delta_{\mu}\right\|_{p q, \omega} \sum_{v=2^{\mu-1}}^{2^{\mu}-2} \mid\left(1-\frac{\sin v t}{v t}\right)^{r-[r]}\left(1-\frac{\sin v h_{1}}{v h_{1}}\right) \ldots\left(1-\frac{\sin v h_{[r]}}{v h_{[r]}}\right) \\
-\left(1-\frac{\sin (v+1) t}{(v+1) t}\right)^{r-[r]}\left(1-\frac{\sin (v+1) h_{1}}{(v+1) h_{1}}\right) \\
\cdots\left(1-\frac{\sin (v+1) h_{[r]}}{(v+1) h_{[r]}}\right) \mid\left\|\sum_{l=2^{\mu-1}}^{v} A_{l}(f, x)\right\|_{p q, \omega}
\end{gathered}
$$




$$
\begin{aligned}
& +\mid\left(1-\frac{\sin \left(2^{\mu}-1\right) t}{\left(2^{\mu}-1\right) t}\right)^{r-[r]}\left(1-\frac{\sin \left(2^{\mu}-1\right) h_{1}}{\left(2^{\mu}-1\right) h_{1}}\right) \\
& \quad \ldots\left(1-\frac{\sin \left(2^{\mu}-1\right) h_{[r]}}{\left(2^{\mu}-1\right) h_{[r]}}\right) \mid\left\|\sum_{l=2^{\mu-1}}^{2^{\mu}-1} A_{l}(f, x)\right\|_{p q, \omega}
\end{aligned}
$$

and by Lemma D

$$
\left\|\sum_{l=2^{\mu-1}}^{v} A_{l}(f, x)\right\|_{p q, \omega} \leq c E_{2^{\mu-1}-1}(f)_{p, \omega}
$$

and

$$
\left\|\sum_{l=2^{\mu-1}}^{2^{\mu}-1} A_{l}(f, x)\right\|_{p q, \omega} \leq C E_{2^{\mu-1}-1}(f)_{p q, \omega} .
$$

Since $x^{r}\left(1-\frac{\sin x}{x}\right)^{r}$ is non decreasing for positive $x$ we have

$$
\left\|\delta_{\mu}\right\|_{p q, \omega} \leq c 2^{2 \mu r} t^{2(r-[r])} h_{1}^{2} h_{2}^{2} \ldots h_{[r]}^{2} E_{2^{\mu-1}-1}(f)_{p q, \omega}
$$

and hence

$$
\begin{aligned}
& \left\|\sigma_{t, h_{1}, h_{2}, \ldots, h_{[r]}}^{r} S_{2^{m-1}}(\cdot, f)\right\|_{p q, \omega} \\
& \leq c t^{2(r-[r])} h_{1}^{2} h_{2}^{2} \ldots h_{[r]}^{2}\left\{\sum_{\mu=1}^{m} 2^{\mu r \gamma} E_{2^{\mu-1}-1}^{\gamma}(f)_{p q, \omega}\right\}^{1 / \gamma} \\
& \leq c t^{2(r-[r])} h_{1}^{2} h_{2}^{2} \ldots h_{[r]}^{2}\left\{2^{\gamma r} E_{0}^{\gamma}(f)_{M}\right\}^{1 / \alpha} \\
& +c t^{2(r-[r])} h_{1}^{2} h_{2}^{2} \ldots h_{[r]}^{2}\left\{\sum_{\mu=2}^{m} \sum_{v=2^{\mu-2}}^{2^{\mu-1}-1} v^{2 \gamma r-1} E_{v-1}^{\gamma}(f)_{p q, \omega}\right\}^{1 / \gamma} \\
& \leq c t^{2(r-[r])} h_{1}^{2} h_{2}^{2} \ldots h_{[r]}^{2}\left\{\sum_{v=1}^{2^{m-1}-1} v^{2 \gamma r-1} E_{v-1}^{\gamma}(f)_{p q, \omega}\right\}^{1 / \gamma} .
\end{aligned}
$$

Therefore we find

$$
\Omega_{r}\left(f, \frac{1}{n}\right)_{p q, \omega} \leq \frac{c}{n^{2 r}}\left\{\sum_{\nu=1}^{n} v^{2 \gamma r-1} E_{v-1}^{\gamma}(f)_{p q, \omega}\right\}^{1 / \gamma}
$$

finishing the proof of Theorem 1.7.

\section{References}

[1] Akgün R., Sharp Jackson and converse theorems of trigonometric approximation in weighted Lebesgue spaces, Proc. A. Razmadze Math. Inst. 152 (2010), 1-18.

[2] Akgün R., Yildirir Y. E., Jackson-Stechkin type inequalities in weighted Lorentz spaces, Math. Inequal. Appl. 18, 4 (2015), 1283-1293. 
[3] Bennet C., Sharpley R., Interpolation of operators. Academic Press, Inc., Boston, MA, 1968.

[4] Berkson E., Gillespie T. A., On restrictions of multipliers in weighted settings, Indiana Univ. Math. J. 52 (2003), 927-962

[5] Chang H. M., Hunt R. A. and Kurtz D. S., The Hardy-Littlewood maximal functions on $L(p, q)$ spaces with weights, Indiana Univ. Math. J. 31 (1982), 109-120.

[6] Dai F., Ditzian Z., and Tikhonov S., Sharp Jackson inequalities, J. Approx. Theory 151 (2008), 86-112.

[7] Ditzian Z., Totik V., Moduli of Smoothness, Springer Ser. Comput. Math. 9, Springer, New York, 1987.

[8] Ditzian Z., Tikhonov S., Ul'yanov and Nikol'skii-type inequalities, J. Approx. Theory 133 (2005), 100-133.

[9] Guven A., Israfilov D. M., Improved Inverse Theorems in Weighted Lebesgue and Smirnov Spaces, Bull. Belg. Math. Soc. Simon Stevin 14, (2007), 681-692.

[10] Gadjieva E. A., Investigation the Properties of Functions with Quasimonotone Fourier Coefficients in Generalized Nikolskii-Besov Spaces (Russian), Authors Summary of Candidates Dissertation, Tbilisi (1986).

[11] Genebashvili I., Gogatishvili A., Kokilashvili V., Krbec M., Weight theory for integral transforms on spaces of homogenous type, Pitman Monographs, 1998.

[12] Jafarov S. Z., The inverse theorem of approximation of the function in SmirnovOrlicz classes, Math. Inequal. Appl. 12 (2012), 835-844.

[13] Kokilashvili V., Krbec M., Weighted inequalities in Lorentz and Orlicz spaces. World Scientific Publishing Co. Inc. River Edge, NJ, 1991.

[14] Kokilashvili V. M., Yildirir Y. E., On the approximation by trigonometric polynomials in weighted Lorentz spaces, J. Funct. Spaces Appl. 8 (2010), 67-86.

[15] Kokilashvili V. M., Yildirir Y. E., On the approximation in weighted Lebesgue spaces, Proc. A. Razmadze Math. Inst. 143 (2007), 103-113.

[16] Ky N. X., An Alexits's lemma and its applications in approximation theory, Functions, Series, Operators (L. Leindler, F. Schipp, J. Szabados, eds.), Budapest (2002), 287-296.

[17] Muckenhoupt B., Weighted Norm Inequalities for the Hardy Maximal Function, Trans. Amer. Math. Soc. 165 (1972), 207-226.

[18] Natanson G. I., Timan M. F., The geometric means of the sequence of the best approximations, (Russian), Vestnik Leningrad Univ. Mat. Mekh. Astronom., 1979, vyp. 4, 50-52. 
[19] Samko S. G., Kilbas A. A. and Marichev O. I., Fractional integrals and derivatives, Theory and applications, Gordon and Breach Science Publishers, 1993.

[20] Yildirir Y. E., Israfilov D. M., Approximation Theorems in weighted Lorentz spaces, Carpathian J. Math., 26 (2010), 108-119.

[21] Yildirir Y. E., Israfilov D. M., Simultaneous and converse approximation theorems in weighted Lebesgue spaces, Math. Ineq. Appl. 14, (2011), 359-371.

[22] Zygmund A, Trigonometric series, Cambridge, 1959.

Department of Mathematics, Faculty of Art-Science,

Balikesir University

10100 Balikesir, Turkey

email: rakgun@balikesir.edu.tr

Department of Mathematics, Faculty of Education,

Balikesir University

10100, Balikesir, Turkey

e-mail: yildirir@balikesir.edu.tr 